\title{
MiRNA-210: A Current Overview
}

\author{
ALBERTO BAVELLONI ${ }^{1}$, GIULIA RAMAZZOTTI ${ }^{2}$, ALESSANDRO POLI ${ }^{3,4}$, MANUELA PIAZZI $^{1,5}$, \\ ENRICO FOCACCIA ${ }^{1,5}$, WILLIAM BLALOCK $^{1,5^{*}}$ and IRENE FAENZA ${ }^{2 *}$ \\ ${ }^{I}$ SC Laboratory of Musculoskeletal Cell Biology, Rizzoli Orthopedic Institute (IOR), Bologna, Italy; \\ ${ }^{2}$ Department of Biomedical and Neuromotor Sciences, University of Bologna, Bologna, Italy; \\ ${ }^{3}$ Istituto Nazionale Genetica Molecolare "Romeo e Enrica Invernizzi", Milan, Italy; \\ ${ }^{4}$ Department of Medical Biotechnology and Translational Medicine, Università degli Studi di Milano, Milan, Italy; \\ ${ }^{5}$ Institute of Molecular Genetics-National Research Council of Italy (IGM-CNR), UOS Bologna, Bologna, Italy
}

\begin{abstract}
RNAs (miRNAs) are a group of highly conserved small non-coding RNAs that were found to enhance $m R N A$ degradation or inhibit post-transcriptional translation. Accumulating evidence indicates that miRNAs contribute to tumorigenesis and cancer metastasis. microRNA-210 has been largely studied in the past several years and has been identified as a major miRNA induced under hypoxia. A variety of miR-210 targets have been identified pointing to its role, not only in mitochondrial metabolism, but also in angiogenesis, the DNA damage response, cell proliferation, and apoptosis. Based on earlier research findings, this review aims to provide a current overview on the involvement of miRNA-210 in biological processes and diseases.
\end{abstract}

The term "RNA processing" refers to several aspects of RNA metabolism including, splicing and alternative splicing, posttranscriptional nucleotide modifications (methylation, pseudouridylation, deamination), folding, cellular localization, transport and degradation $(1,2)$. Among functional RNA molecules non-coding RNAs work without being translated into a protein. Non-coding RNAs include transfer RNAs (tRNAs) and ribosomal RNAs (rRNAs), as well as RNAs such

This Article is freely accessible online.

*These Authors contributed equally to this work.

Correspondence to: Irene Faenza, Department of Biomedical and Neuromotor Sciences, University of Bologna, Bologna, Italy. Tel: +393392287797, e-mail: irene.faenza2@unibo.it and William Blalock, SC Laboratory of Musculoskeletal Cell Biology, Rizzoli Orthopedic Institute (IOR), Bologna, Italy. Tel: +39 051626770, e-mail:william.blalock@cnr.it

Key Words: microRNA, miR-210, hypoxia, cancer, disease, review. as snoRNAs, microRNAs, siRNAs, snRNAs, exRNAs, piRNAs and scaRNAs and the long ncRNAs including for example Xist and HOTAIR (3).

microRNAs (miRNas) are members of a larger class of non-coding RNAs that control gene expression and regulate a wide array of biological processes by targeting messenger RNAs (mRNA) and inducing translational repression or RNA degradation $(4,5)$. Cells naturally produce small non-coding RNAs, referred to as miRNAs, from genome encoded sequences, which contain their own promoter elements. The result of their transcription is on average a 70nt-long transcript with a stem-loop structure. miRNAs regulate gene expression by binding to the mRNA of the target genes and direct their post-transcriptional repression. Some miRNAs show clear complementarity to their target mRNA and lead to their degradation. Others pair imperfectly with the 3'UTR of the target mRNA and inhibit translation (protein production) (6). Precursor miRNAs transcripts are mainly processed by the nuclear ribonuclease RNase III, Drosha and then exported to the cytoplasm, by the karyopherin-exportin 5 complex. Once in the cytoplasm the pre-miRNAs are processed into 21-22 nucleotide long miRNAs by the RNAase activity of Dicer.

The miRNA duplex then associates with the RNA-induced silencing complex (RISC) and its processing is mediated by the argonaute (AGO) family, in the presence of other cofactors such as PACT (also known as PRKRA). miRNAs guide RISC to target messages that are subsequently cleaved or translationally silenced. miRNAs regulate gene expression by binding to the mRNA of the target genes and direct their post-transcriptional repression. Thus, miRNAs represent an important piece of the puzzle that is gene expression regulation. Consequently, aberrant expression of a single miRNA can dramatically and simultaneously alter the translation of several genes within a cell, thereby altering the 
cell phenotype. Modifications of miRNAs can alter their target specificity or function. miRNA editing may represent a fine tuning process in miRNA biogenesis at different steps, resulting in variations of target mRNAs and providing a regulatory control within the complex network of RNAmediated gene functions. The miRNA biogenesis pathway is targeted by the RNA editing proteins, ADARs $(7,8)$.

Several studies have reported links between alterations of miRNA homeostasis and pathological conditions. Therefore, as a result, aberrant miRNA expression affects several aspects of cellular function from proliferation and differentiation to apoptosis, associating miRNA alteration to pathology. In addition, miRNA deficiencies or excesses have been correlated with a number of clinically important diseases ranging from cancer to myocardial infarction (9).

A growing body of experimental evidence suggests a clinical relevance for microRNA (miR)-210. There are two versions of miR-210, namely, miR-210-3p and miR210-5p. miR-210-3p is the guide-strand that integrates into the RISC, whereas miR-210-5p is the passenger-strand that is inactivated through degradation (10). miR-210 is up-regulated in most solid tumors, and its levels correlate with a negative clinical outcome. It has been associated with a variety of functionally important targets involved in cancer but also in cell cycle regulation, cell survival, differentiation, angiogenesis, as well as in metabolism (11-13). In this review, we focus on the following current areas of research regarding miR-210: its involvement in important biological processes, in hypoxic conditions and in cancer.

\section{Biological and Cellular Processes}

miR-210 is involved in numerous biological processes almost throughout the human body. miR-210 is a regulator of several cellular functions, dependent or independent on hypoxia; among these, neurogenesis, which is a complex process of many steps. In brain injuries, neurogenesis is characterized by intense proliferation and migration of endogenous neural stem cells (NSCs) that peaks several days after injury, followed by a prolonged period of differentiation, survival, and integration. Recent studies provide contradictory reports on whether miR210 action promotes (14) or suppresses (15) post-ischemic neurogenesis in vivo. The mechanisms underlying the decreased rates of NSC proliferation induced by miR-210 suppression and the accompanying metabolic changes have been recently investigated. miR-210 has a role in regulating mitochondrial metabolism (16); however, it was also found that miR-210 inhibition enhanced mitochondrial oxidative metabolism in NSC cultures. NSC proliferation is attenuated by miR-210 inhibition induced by treatment with media preconditioned using pro-inflammatory activated microglia (CM). miR-210 inhibition during inflammatory CM injury effectively protects mitochondrial function, as evidenced by increased levels of cytochrome c oxidase and aconitase activities. A current study evidenced that miR-210 inhibition enhanced mitochondrial oxidative metabolism in NSC cultures. In particular, miR-210 inhibition significantly attenuated NSC proliferation and glycolytic activity at the early stages of differentiation. Mitochondrial protection induced by miR-210 inhibition thus potentially provides a new strategy to protect neurogenesis in inflammatory settings (17).

Another biological complex process is angiogenesis. This process involves the migration, growth, and differentiation of endothelial cells to form new blood vessels from pre-existing ones. Angiogenesis involves interactions between endothelial cells and the microenvironment. A hypoxic microenvironment can induce angiogenesis, up-regulating numerous proangiogenic factors, of which vascular endothelial growth factor (VEGF) is the best example. When miR-210 is overexpressed in human umbilical vein endothelial cells (HUVECs), the ability of those cells to form blood vessels becomes more significantly pronounced than in cells with normal levels of expression (18). Interestingly, miR-210 expression is correlated closely with VEGF expression, hypoxia, and angiogenesis in breast cancer patients, indicating a possible role for miR-210 in tumor angiogenesis (19). In endothelial cells, modulation of miR-210 expression and/or activity may be a viable strategy to control angiogenesis in that the expression of ephrin-A3 (Efna3), a protein involved in the development of the cardiovascular system and in vascular remodeling, is inhibited by miR-210 expression (18). Importantly, extracellular vesicles (EVs), membrane bound structures containing RNAs, proteins, DNA, miRNA and bioactive lipids, secreted by mesenchymal stem cells (MSCs) promote angiogenesis in ischemic myocardium through a miR210-Efna3 dependent mechanism. In fact, this effect is dependent on the enrichment of miR-210 in MSCs-EVs (20). miR-210 is involved in angiogenesis after cerebral ischemia, as its expression level is increased in postischemic brain tissue. When miR-210 is overexpressed in HUVECs, Notch 1 protein levels increase which, in turn, regulates angiogenesis and the maturation of vasculature in the post-ischemic brain tissue (21). miR-210 is also involved in pro-angiogenic effects during acute colitis (22). Both in vitro and in vivo studies reveal that high levels of miR-210 sustain angiogenesis in hepatocellular carcinoma by targeting fibroblast growth factor receptor-like 1 (FGFRL1) expression (23). Furthermore Xiao F. et al. demonstrated that miR-210 expression and its pro-angiogenesis function were inhibited by the heparan sulfate mimetic WSS25, providing further insight into potential new drug development in terms of miRNAs (24).

miR-210 is also involved in proliferation and apoptosis. Elevated expression of miR-210 induces proliferation and decreases apoptosis in glioblastoma multiforme (GBM) cells by targeting the regulator of differentiation 1 (ROD1), which 
is involved in GBM progression (25). A pro-apoptotic function that miR-210 has at normal oxygen levels and an anti-apoptotic effect under hypoxic conditions has also been described (26). In order to define a new strategy for protecting against endothelial injury in atherosclerosis, a recent study was conducted in endothelial cells examining the effect of miR-210 under oxidative stress. miR-210 levels are high in HUVECs treated with $\mathrm{H}_{2} \mathrm{O}_{2}$. Moreover, miR-210 acts as a pro-survival and anti-apoptotic factor in HUVECs under oxidative stress by reducing ROS generation and downregulating the CASP8AP2 pathway (27).

Recently, many miRNAs have been reported to play roles in erythropoiesis regulation in that miR-210 is expressed predominantly during erythrocyte development in immature progenitors until development into mature erythrocytes (28). In addition, miR-210 is up-regulated in status epilepticus. In a study aimed at identifying the typical characteristic of a status epilecticus, such as decreased GABAAR $\alpha 1$ expression, in which epileptic activity was induced in rats by injecting $\mathrm{LiCl}$ and pilocarpine, the authors found that epileptic activity led to apoptosis of the hippocampal neurons and that interestingly a miR-210 inhibitor attenuates the apoptosis induced by epileptic activity (29). Duan et al. characterized the expression of miR-210 in the reproduction system, investigating the potential role of miR-210 in spermatogenesis and cryptorchidism, the relationship between miR-210 and Nuclear Receptor Subfamily 1, Group D, Member 2 (NR1D2) in the testicular embryonic carcinoma cell line NTERA-2 (NT-2), and the potential targets of miR210 that may participate in testis development. Prediction algorithm miRanda analysis revealed that the 3'-UTR of NR1D2 mRNA presents one presumptive miR-210 binding site. In this system miR-210 negatively modulates the expression of NR1D2, at the transcriptional and translational levels, by directly binding to the seed sequence of the NR1D2 3' -UTR. Furthermore, they determined the expression of miR-210 at different stages of cryptorchidism, establishing a mouse model of cryptorchidism and finding that miR-210 expression increased significantly at 14 and 21 days after surgery. Finally, they demonstrated that miR-210 acts as an upstream regulator of NR1D2 function in human cryptorchidism (30).

\section{Hypoxic Condition}

Hypoxia is the most common neoplastic microenvironment in solid tumors in which oxygen concentration is reduced markedly. Hypoxia, induces the expression of several miRNAs, which in turn play important roles in the adaptive response to low oxygen in tumors (19). Most studies suggest a direct connection between miR-210 expression and hypoxia (31-33) and in particular, carrying the HIF-1 $\alpha$-binding sites in its promoter, miR-210 is significantly up-regulated in both normal and transformed cells $(34,35)$. Hypoxia-Inducible Factors (HIFs) are well-documented master regulators of the hypoxia response. Indeed, in tumor microenvironments, the HIF-1 $\alpha$ oxygen-dependent degradation pathway is halted by increased levels of HIF-1 $\alpha$ (36). Several studies have reported miR-210 as one of the highly up-regulated miRNAs in hypoxic cells and have demonstrated its importance for cell survival $(18,37)$. Recently, a miR-210 hypoxic circuit has been described (38) in which miR-210 regulates HIFs. In particular, Kelly et al. identified a new regulator of HIF-1, glycerol-3-phosphate dehydrogenase 1-like (GPD1L), which is regulated by the HIF-1-inducible microRNA miR-210. In fact, induction of miR-210 by HIF-1 causes a decreased in GPD1L protein expression, which in turn results in increased HIF-1 stability. Under normal physiological conditions, GPD1L acts to increase the activity of the prolyl-hydroxylase domain isoforms (PHDs), which ensures HIF-1 proline hydroxylation, leading to HIF-1 degradation by the proteasome. Overexpression of miR-210 resulted in increased HIF-1 accumulation during hypoxia through decreased expression of GPD1L protein due to miR-210 targeting of the GPD1L mRNA 3' UTR. When HIF-1 protein expression is low, the level of miR-210 is low as well, and, as a consequence, GPD1L expression is turned-on. As oxygen levels decrease, HIF-1 protein and transcriptional activity increase, leading to accumulation of miR-210. As a consequence, expression of GPD1L is down-regulated and the PHDs are inactivated, resulting in increased HIF-1 protein. This mechanism consists in a positive feedback circuit where miR-210 induces and maintains the HIF-1 protein level. This hypoxic circuit can be perturbed by inhibition of miR-210 (38). Moreover, in this regard, Costales MG et al. reported that the miR-210 hypoxic circuit is modulated by a small molecule Targapremir-210, which binds to miR-210's Dicer processing site. They demonstrated that this interaction inhibits production of mature miR-210, which leads to apoptosis of triple negative breast cancer cells only under hypoxic conditions, as well as to inhibition of tumorigenesis in a mouse xenograft model of hypoxic triple negative breast cancer (39). In addition, as the level of miR-210 relies on the level of HIF-1 $\alpha$, high expression of miR-210 in tissues and serum has become a predictive marker for tumor hypoxia (40, 41). Functional studies indicate that miR-210, in response to hypoxic stress, down-regulates the DNA repair factor RAD52 (42). miR-210 expression is up-regulated by hypoxia in diverse cancer cell lines, such as colon and breast carcinoma and esophageal adenocarcinoma $(35,43)$. miR-210 can negatively regulate mitochondrial respiratory activity and increase ROS generation. ISCU (iron-sulfur cluster scaffold homolog) and COX10 (cytochrome c oxidase assembly protein) genes, components involved in the mitochondrial electron transport chain and the tricarboxylic acid (TCA) cycle, have been described as targets of miR-210 (16). More 
recently, it has been demonstrated that HIF-1 $\alpha$, CAIX (carbonic anhydrase), miR-210 and ISCU represent a relevant signalling axis in oropharyngeal small-cell carcinomas (SCCs) exposed to hypoxia. This coordinated association, related to poor prognosis, is a finding with important implications for the development of metabolic-targeting therapies against hypoxia (44). In hypoxic human hepatoma cells, miR-210 down-regulation significantly suppresses cell viability, induces cell cycle arrest, increases apoptosis and enhances radiosensitivity through directly targeting the hypoxia stress response cell death inducer mitochondrionassociated 3 (AIFM3) (45).

Molecular evidence for hypoxia was demonstrated in Preeclampsia (PE) which is a unique multisystem disease of human pregnancy characterized by maternal hypertension and proteinuria placentas. In placenta tissue isolated from preeclamptic patients, where hypoxia has a causal role, miR-210 expression is increased $(46,47)$. It has been demonstrated that overexpression of miR-210 mediates mitochondrial dysfunction in the placenta of women who present complications of PE during pregnancy (48). Oddly enough, it appears that mitochondrial dysfunction has a crucial role in the pathogenesis of PE disease in that placental hypoxia, due to the presence of HIF-1 in pre-eclamptic tissues, may play a causal role in the mitochondrial abnormalities observed (48). Recently Luo R. et al. described a crucial role of miR-210 in human placenta; they demonstrated that miR-210 regulates trophoblast function in part by targeting thrombospondin type I domain containing 7A (THSD7A) (49). Moreover, Zhang Y et al. described that higher levels of mir-210 are found in patients with PE and hypoxia-treated trophoblast cells and identify a new transcriptional mechanism, independent from the well-known regulator HIF-1 $\alpha$, in which NF-kB transcriptional factor $\mathrm{p} 50$, regulates miR-210 expression (50). Interestingly, miR-210 is also involved in non-hypoxic pathways related to abnormal angiogenic and metabolic alterations noted in PE. In placental tissues, miR-210 is associated weakly with the expression of HIF-1 $\alpha$, presenting evidence against a direct relationship between these two molecules, at least in this context. In contrast, miR-210 is strongly correlated with sFlt1 expression during PE, suggesting that both these pathways may have a common upstream regulator (51). Protein tyrosine phosphatase, nonreceptor type 2 (PTPN2) is a direct target of miR-210 (52). Placental expression of miR-210 is up-regulated in pregnancies complicated with $\mathrm{PE}$ in comparison to normal pregnancies. This increased level of miR-210 is related to down-regulation of PTPN2 mRNA, indicating a direct role in the pathogenesis of PE disease (53).

Hypoxia is an important feature in tumors with malignant phenotypes and hypoxic tumors may communicate with surrounding tumor and non-tumor cells through exosomes to induce phenotypes that are more malignant. As cell-derived vesicles (30-100 $\mathrm{nm}$ in diameter) secreted by cells, exosomes contain many functional proteins, mRNAs, and miRNAs, and play a role in intercellular communication. They are capable of diffusing to neighboring cells or being transported to distant anatomic locations where they can transduce signals or information in specific recipient cells $(54,55)$. Tumor cells of many different cancer types have been shown to secrete exosomes in greater amounts than normal cells, thus allowing the transfer of tumor-associated signaling molecules, including miRNAs, via fusion of the exosome with the target cell membrane. miRNAs from hypoxic cancer cells, spread to adjacent cancer cells in the tumor microenvironment via exosomes and influence tumor progression (56). In this regard it has been described that hypoxic cancer cells release exosomes with high levels of miR-210 $(57,58)$. Jung et al. investigated systemic transfer of exosomal miR-210 in the blood circulation and observed a significant increase in the amount of miR-210 from exosomes isolated from the serum of hypoxic tumor bearing mice, indicating that miR-210 from circulating exosomes in the serum can be used as a potential biomarker for hypoxic tumors. They also demonstrated that exosomal miR-210 from hypoxic cancer cells can be transferred to various types of recipient cells, such as epithelial cells, immune cells, and mesenchymal stem cells (54). Patients with breast cancer present increased levels of circulating miR-210; thus, circulating miR-210 may be a potential biomarker of tumor presence and therapeutic response in breast cancer (59). Moreover, hypoxia-inducible circulating miR-210 contributes to metastasis in colorectal cancer (CRC) (60), and it has recently been described as a potential non-invasive marker for diagnosis and prognosis of colorectal cancer (CRC) (61). Given the several different roles that miR-210 plays in numerous aspects of cellular function, it is also noteworthy to mention that hypoxia induces K562 and $\beta$-thalassemic erythroid progenitor cell differentiation, and this induction is at least in part mediated by miR-210, which, in turn, modulates a linkage between erythropoiesis and hypoxia (62). The miR-210 is associated with the elevated level of fetal $\gamma$-globin in mithramycininduced K562 cells (63), and in this regard recent data confirmed that phospholipase $\mathrm{C}-\beta 1$ can regulate miR-210 levels through the PKC $\alpha$ signaling pathway $(64,65)$. More recently, in order to assess erythroid and tissue condition to chronic anemia and hypoxia, red blood cells and plasma of $\beta$-thalassemia patients were analyzed for the presence of miR-210. The data indicated that miR-210 expression levels in $\beta$-thalassemia/HbE patients were increased in both red blood cells and plasma. The increased expression of miR-210 in red blood cells was inversely associated with hemoglobin levels and hematocrit (66).

Recent research has indicted that expression of HIF-1 $\alpha$ and miR-210, in response to a hypoxic condition, is significantly up-regulated in epithelial ovarian cancer (EOC) samples as 
well as EOC cell lines, indicating that high levels of miR-210 in EOC specimens are associated with tumor stage and the post-operative residual tumor size. Results also demonstrated that up-regulated miR-210 enhanced the proliferative capacity and colony formation of EOC cells in vitro and significantly silenced tyrosine-protein phosphatase non-receptor type 1 (PTPN1), blocking its pro-apoptotic effect (67). Moreover, an additional study shows that miR-210 supports ovarian cancer cell mobility by acting as a modulator of E-cadherin expression and consequently on epithelial-mesenchymal transition (EMT) (68). miR-210 expression level is induced in normoxic myoblasts upon myogenic differentiation both in vitro and in vivo. This regulation occurred at the transcriptional level with a HIF1-alpha dependent mechanism. In fact, a characterization of the promoter region of miR-210 show that HIF-1alpha is responsible for induction of miR-210 during $\mathrm{C} 2 \mathrm{C} 12$ myogenic differentiation. The promoter region identified is a functional HRE site, localized at-459bp from mature mouse miR-210 (69). Additional regulators of miR-210 have been explored. It is worth noting that Akt increases miR-210 levels through a HIF-independent mechanism in hypoxic cardiomyocytes (70). Among the potential miR-210 regulators, a conserved NF-kB binding site has also been mapped in the promoter region (50). Strategies targeting to correct the aberrantly expressed miR-210 might open up a new therapeutic avenue to hypoxia and in this regard Yang W. et al. explored the possibility of miR-210 as an effective therapeutic target to hypoxic glioma stem cells (GSCs) employing a lentiviral-mediated anti-sense miR-210 gene transfer technique to knockdown miR-210 (71).

\section{miR-210 and Cancer}

miR-210 has been extensively studied in cancer progression (72), and it is known that miR-210 generally exhibits oncogenic properties, as it is frequently elevated in several cancers including breast, lung, head and neck, pancreatic cancer, or glioblastoma(35, 73-79). The overexpression of miR-210 in breast cancer is correlated with a poor prognosis, being associated with aggressiveness and shorter time to distant metastasis (80-82). miR-210 was shown to be upregulated in triple-negative breast cancer (TNBC), defined by the lack of estrogen receptor, progesterone receptor, and HER2 gene amplification, compared with estrogen positive breast cancer $(83,84)$. miR-210 is overexpressed in numerous cancers including breast (85) and the induction of miR-210 is a consistent characteristic of the hypoxic response in both normal and transformed cells as we described in the section before but Bar et al. interestingly suggested that miR-210 expression is regulated by a mechanism independent of HIF1alpha in infiltrated immune cells of the TNBC (86). Moreover, the cellular localization of miR-210 has recently been shown through in situ hybridization (ISH) for miRNA, a technique that allows the precise histological localization of miRNAs in situ. Bar et al. performed ISH to visualize miR210 localization in TNBC samples and showed that miR-210 is expressed not only in epithelial cancer cells but also in the tumor microenvironment (TME), particularly in inflammatory cells (85). miR-210 is also involved in mitochondrial dysfunction in various types of cancer (87). The expression profiling of cells overexpressing miR-210 show enrichment of transcripts related to cell death and mitochondrial dysfunction, including several subunits of the electron transport chain (ETC) complexes I and II (88).

miRNAs can be secreted from cells, be present in the circulation, or be taken up by other cells. The presence of circulating miRNAs has been demonstrated in various disease conditions, and the profiles vary with the degree of disease progression. Since unprotected miRNAs are sensitive to degradation by RNases present in the blood, stably existing circulating miRNAs are thought to be packaged in microvesicles, exosomes and apoptotic bodies or bound to RNA-binding protein such as argonaute 2 or lipoprotein complexes, and are thus highly stable $(89,90)$. Recent evidence has emerged that circulating miRNAs potentially derive directly from tumor tissue, thereby correlating with tumor progression and recurrence (91). Circulating miRNAs in blood samples could be a diagnostic tool for patients with cancer (92). In this regard, Zhao et al. described that the circulating miR-210 is significantly elevated in patients with clear cell or conventional renal cell carcinoma (ccRCC) compared with healthy controls. In addition, the expression level of miR-210 greatly decreases post-operatively, suggesting that serum miR-210 could start-out partially from the primary renal tumors (93). More recently, the amount of miR-210 has been analyzed in cell-free urine as a tool for liquid biopsy in ccRCC; the level of circulating miR-210 in urine is significantly higher in patients with ccRCC than in control subjects (94). In clinical ccRCC tissues miR-210-3p is generally up-regulated (95). In a very recent study Yoshino $\mathrm{H}$ et al. targeted miR-210-3p and utilized the CRISPR/Cas9 gene editing system to deplete miR-210-3p in RCC cell lines. Its down-regulation resulted in increased tumorigenesis, both in vitro and in vivo. They also found that found that miR-210$3 p$ down-regulation resulted in dramatic up-regulation of Twist-related protein 1 (TWIST1) which is the key target of miR-210-3p in this system suggesting renal cell carcinoma progression is promoted by TWIST1 suppression mediated by miR-210-3p (96).

Furthermore, serum miR-210 was up-regulated in patients with bladder cancer (BC), and serum levels of miR-210 increased with advancing stage and grade $(97,98)$. An increase in miR-210 expression occurs also in osteosarcoma (OS) tissues and miR-210 up-regulation demonstrates a strong correlation with tumor aggressive progression of pediatric osteosarcoma. Moreover, this up-regulation is correlated with 
large tumor size, chemoresistance, metastasis, and poor prognosis of OS patients (99). An analysis carried-out in a group of 51 samples, including adrenocortical carcinoma, adrenocortical adenoma and normal adrenal cortex, showed the expression of 15 microRNAs up-regulated in carcinoma as compared to normal tissue; among these was miR-210 (100). MiR-210 is significantly up-regulated in three human osteosarcoma cell lines, U2OS, Saos-2 and MG63, as well as in the human osteoblast cell line hFOB. miR-210 has a crucial role in OS development and progression. Down-regulation of miR-210 can inhibit cell proliferation, clonogenicity, migration, invasion, as well as induced $\mathrm{G}_{1}$ arrest and cell apoptosis in vitro, as well as suppress tumor growth in vivo. Transfection with miR-210 inhibitor inhibits cell migration and invasion of MG63 cells and, in addition, suppressed tumor growth of OS in nude mice (101). miR-210 participated in the dedifferentiation of OS cells into OS stem cells (OSCs), in fact, miR-210 positively participates in TGF- $\beta 1$ - and hypoxiainduced OS cell dedifferentiation, thus inhibiting miR-210 and significantly reducing formation of OSCs (102). miR-210 functions not only in tumorigenesis, but it can also act as a tumor suppressor, inhibiting tumor initiation in a small number of cancer cells including ovarian cancer, esophageal squamous cell carcinoma (ESCC) and laryngeal squamous cell carcinoma (LSCC) $(35,36)$. In particular, expression of miR210 is down-regulated in esophageal squamous cell carcinoma (ESCC) cell lines as well as in clinical samples. In these models miR-210 induces cell cycle arrest and apoptosis in vitro, thus inhibiting the proliferation of cancer cells. In these models data suggest that down-regulation of miR-210 might play an important role in the proliferation of ESCC (103). It has also been described that in laryngeal squamous cell carcinoma (LSCC) high expression of miR-210 inhibits the proliferation via inducing cell cycle arrest and apoptosis by the targeting of FGFRL1 (104). Giannakakis et al. has shown that miR-210 is deleted in $50 \%$ of ovarian cancer cell lines and $64 \%$ of ovarian cancer samples tested, demonstrating an extensive loss of heterozygosity of an important region where frequent loss of heterozygosity was observed also in lung and breast cancer, highlighting miR-210 as a candidate tumor suppressor gene. They found a statistically significant correlation between the expression of mature miR-210 and gene copy number in ovarian cancer showing that genetic alterations within the miR-210 gene directly affect its expression level in ovarian cancer (105). Over-expression of ectopic miR-210 could function as a tumor suppressor because it attenuates cancer cell proliferation through down-regulating E2F3, fibroblast growth factor receptor-like 1, Homeobox protein Hox-A1 (HOXA1), Homeobox protein Hox-A9 (HOXA9) and Max-binding protein (MNT) $(34,103,106)$.

Recently it has been shown in CNE nasopharyngeal carcinoma cells that miR-210 has an inhibitory role on tumor formation, partially due to mitotic disturbances, as the inhibition of endogenous miR-210, under conditions where it is expressed, rescues cell proliferation, particularly in hypoxic cells (107). In renal carcinoma cell lines miR-210 is constitutive up-regulated under normoxia and this event is correlated to HIF-1 accumulation. Moreover, miR-210 overexpression induced centrosome amplification in both renal carcinoma cells and an immortalized kidney cell line and affects multiple genes associated with cell cycle regulation. In this regard, miR-210 controls $\mathrm{G}_{2} / \mathrm{M}$ transition and is involved in mitotic progression altering the expression of Plk1, Bub1B, cyclin F, Pds5B and Fam83D. In addition, miR-210 is involved in centrosome replication in $\mathrm{S}$ phase by targeting the expression of E2F3. In fact, miR-210 overexpression suppresses the expression of E2F3 and deregulates the centrosome replication cycle, perhaps causing centrosome amplification and aneuploidy (108). Recently, it was discovered that miR-210 regulates sensitivity towards the anticancer agent 1'S-1'-acetoxychavicol acetate (ACA), a natural compound which is able to induce cytotoxicity in various cancer cells, including cervical cancer cells. In fact, miR-210 expression is down-regulated after treatment with ACA in two cervical cancer cell lines leading to a decrease in cell proliferation and apoptosis through a mechanism involving SMAD4, which was identified as a putative target of miR-210 by miRNA target prediction programs (109). miRNA-210, is highly expressed in human gliomas and confers a poor prognosis in glioma patients (110). Furthermore, an increase in miR-210 expression directly correlated with the histopathological grade of astrocytic tumors (111). Recently, an innovative approach to target miR-210 in vivo has been developed. Li Y. et al. provide a strategy using three-layered polyplex with folic acid as a targeting group to systemically deliver miR-210 into breast cancer cells, which results in breast cancer growth being inhibited. The solution of the polyplex is injected in the body of mice via tail intravenous injection and polyplex flows in the blood vessel systemically. The polyplex has good selectivity on breast cancer cells due to cell targeting derived from the recognition of folic acid to overexpressed folate receptor in the cell membrane of breast cancer (112).

Therefore, unusual expression of miR-210 may link to cancer as it has been described to have a prediction value for pancreatic cancer or to be present in considerably higher levels in sputum of lung cancer patients than cancer-free individuals. In this regard $\mathrm{Lu}$ et al. conducted a meta-analysis to evaluate the diagnostic performance of miR-210 in cancer detection. The mechanism of miR-210 abnormally expressed in cancer is not completely understood; more scientific and technological methods should be used in future basic research to provide better understanding of biological roles of miR-210 in cancer, hence lightening up the diagnostic or prognostic value of miR-210. A better understanding of the mechanism of miR-210 regulation expression may achieve optimized therapy. As we mentioned above HIF-1 $\alpha$ binds directly to a 


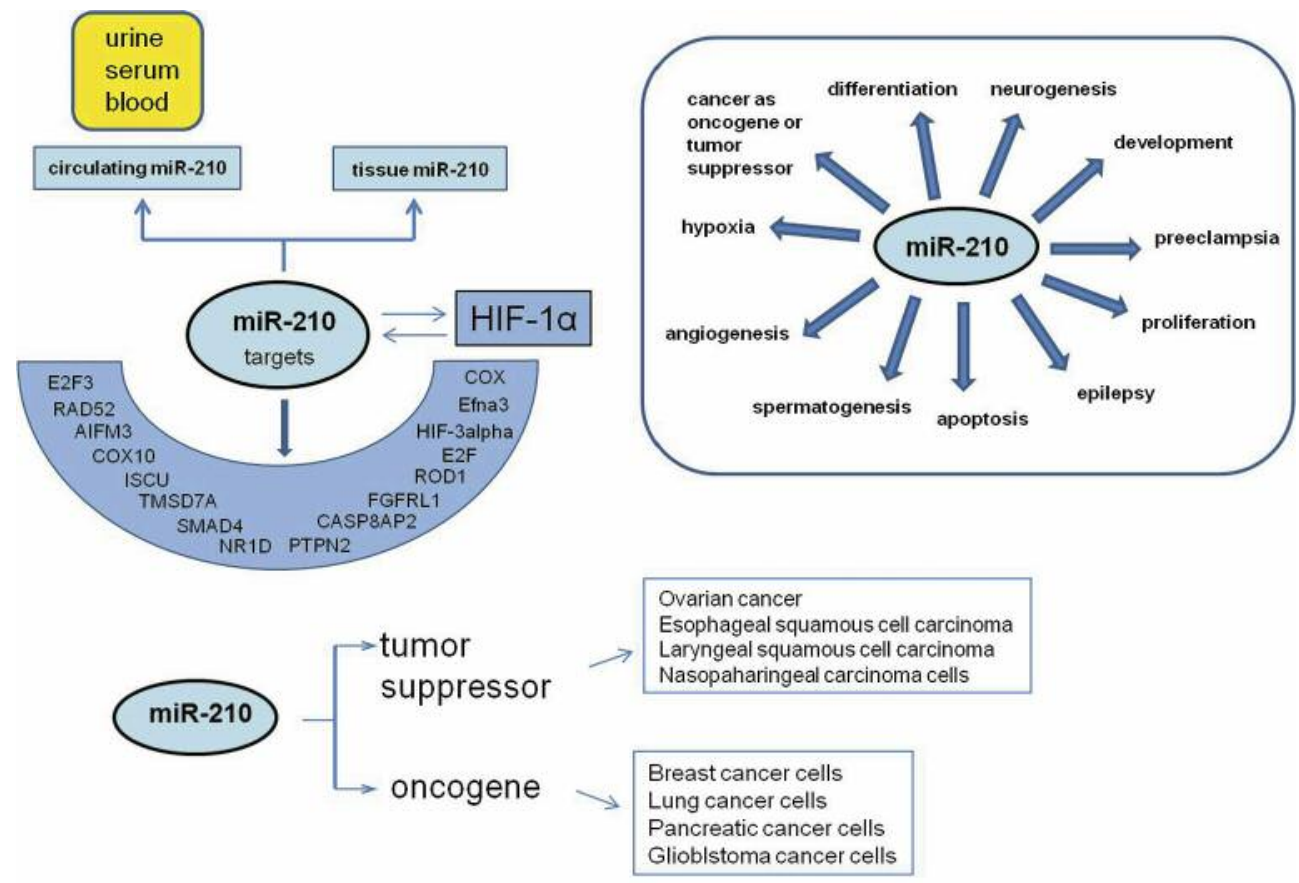

Figure 1. Identified miR-210 target genes implicated in several different biological process and cellular function, indicating a complex functional network regulated by miR-210. MiR-210 could be present in normal or pathological conditions. It could also be released by the cells and circulates in the body fluids of healthy and diseased patients. miR210 can act as oncogene or tumor suppressor.

highly conserved HRE on the proximal miR-210 promoter (34) but additionally, other transcription factors have been described to drive miR-210 expression such as Oct2/4, E2F, NFkB and PPAR $\gamma$ (113-116). In normal and transformed cells it has been shown that miR-210 is up-regulated through protein kinase B (Akt)- and p53-dependent pathway (117). While miR-210 has garnered interest as a possible target in prediction, prognosis and treatment of cancer, further work is required to improve its role and effect.

\section{Conclusion}

On the basis of the experimental evidence summarized, miRNA-210 is one of the most widely studied miRNAs thus far. In addition to its involvement in sophisticated regulation of numerous biological processes, miR-210 has also been shown to be associated with the development of different human diseases and it is regarded as the most important hypoxamir (Figure 1).

In a comparison of microRNA regulation between insects and vertebrates results that certain microRNAs appear to be conserved between flies and mammals. One of these microRNAs is just miR-210. In flies miR-210 had over 70 predicted target genes most of which are evolutionarily conserved and have a known role in Drosophila oogenesis.
This means that miR-210 could be candidate for genes that mediate clade-specific differences in gene expression, and could play an important role in shaping the diversity of life (118). In recent years, emerging data have provided evidence and clarified the role of miR-210, its biological functions and involvement in pathological states, suggesting that miR-210 possess high potential for anticancer therapeutic development. The increasing literature exploring the role of circulating miR210 has clarified its potential as a promising biomarker for early detection, diagnosis and prognosis. Measuring circulating miR-210 levels could be a non-invasive method for early cancer detection. Even if, to date, most studies appear to be preliminary it seems that miR-210 will be a good target for drug development. Overall, it is necessary to underline that miR-210 has a diverse effect on multiple system and this fact limits the use of miR-210 as a therapeutic target. To further expand the knowledge of miR-210's actions may lead to novel diagnostic and therapeutic approaches. Further investigations are also needed to achieve more insight on the regulation of miR-210 expression to facilitate therapeutic-clinical applications in human diseases.

\section{Conflicts of Interest}

The Authors declare no conflicts of interest. 


\section{Acknowledgements}

This work was supported by a Leukemia Research Foundation Grant 2014 and an Italian Association for Research on Cancer (AIRC) Investigator Grant (IG 2015) to WLB, a Fondazione del Monte grant to IF and the $5 \times 1000$ Fund 2013/2014 to the SC Laboratory of Musculoskeletal Cell Biology, Rizzoli Orthopedic Institute (IOR).

\section{References}

1 Blalock WL, Piazzi M, Bavelloni A, Raffini M, Faenza I, D'Angelo A and Cocco L: Identification of the PKR nuclear interactome reveals roles in ribosome biogenesis, mRNA processing and cell division. J Cell Physiol 229: 1047-1060, 2014.

2 Blalock W, Piazzi M, Gallo A, Bavelloni A, Focaccia E and Faenza I: RNA processing and ribosome biogenesis in bone marrow failure disorders. Rna Dis 4: 1-17, 2017.

3 Inamura K: Major tumor suppressor and oncogenic non-coding RNAs: clinical relevance in lung cancer. Cells 6: 12, 2017.

4 Adams B, Kasinski A and Slack F: Aberrant regulation and function of MicroRNAs in cancer. Curr Biol 24: R762-R776, 2014.

5 Croce CM and Calin GA: miRNAs, cancer, and stem cell division. Cell 122: 6-7, 2005.

6 Macfarlane L-A and Murphy PR: MicroRNA: Biogenesis, function and role in cancer. Curr Genomics 11: 537-561, 2010.

7 Gallo A: RNA editing enters the limelight in cancer. Nat Med 19: 130-131, 2013.

8 Mannion N, Arieti F, Gallo A, Keegan L and O'Connell M: New insights into the biological role of mammalian ADARs; the RNA Editing Proteins. Biomolecules 5: 2338-2362, 2015.

9 Ha T-Y: MicroRNAs in human diseases: from cancer to cardiovascular disease. Immune Netw 11: 135-154, 2011.

10 Bartel DP: MicroRNAs: genomics, biogenesis, mechanism, and function. Cell 116: 281-297, 2004.

11 Paul P, Chakraborty A, Sarkar D, Langthasa M, Rahman M, Bari M, Singha RS, Malakar AK and Chakraborty S: Interplay between miRNAs and human diseases: a review. J Cell Physiol, 2017. doi: $10.1002 /$ jcp.25854. [Epub ahead of print]

12 Rupaimoole R and Slack FJ: MicroRNA therapeutics: towards a new era for the management of cancer and other diseases. Nat Rev Drug Discov 16: 203-222, 2017.

13 Ren C-X, Leng R-X, Fan Y-G, Pan H-F, Wu C-H and Ye D-Q: MicroRNA-210 and its theranostic potential. Expert Opin Ther Targets 20: 1325-1338, 2016.

14 Zeng L, He X, Wang Y, Tang Y, Zheng C, Cai H, Liu J, Wang $\mathrm{Y}, \mathrm{Fu} \mathrm{Y}$ and Yang G-Y: MicroRNA-210 overexpression induces angiogenesis and neurogenesis in the normal adult mouse brain. Gene Ther 21: 37-43, 2014.

15 Ma Q, Dasgupta C, Li Y, Bajwa NM, Xiong F, Harding B, Hartman R and Zhang L: Inhibition of microRNA-210 provides neuroprotection in hypoxic-ischemic brain injury in neonatal rats. Neurobiol Dis 89: 202-212, 2016.

16 Chen Z, Li Y, Zhang H, Huang P and Luthra R: Hypoxiaregulated microRNA-210 modulates mitochondrial function and decreases ISCU and COX10 expression. Oncogene 29: 43624368, 2010.

17 Voloboueva LA, Sun X, Xu L, Ouyang Y-B and Giffard RG: Distinct effects of miR-210 reduction on neurogenesis: increased neuronal survival of inflammation but reduced proliferation associated with mitochondrial enhancement. J Neurosci 37: 3072-3084, 2017.

18 Fasanaro P, D’Alessandra Y, Di Stefano V, Melchionna R, Romani S, Pompilio G, Capogrossi MC and Martelli F: MicroRNA-210 modulates endothelial cell response to hypoxia and inhibits the receptor tyrosine kinase ligand ephrin-A3. J Biol Chem 283: 15878-15883, 2008.

19 Huang X and Zuo J: Emerging roles of miR-210 and other noncoding RNAs in the hypoxic response. Acta Biochim Biophys Sin (Shanghai) 46: 220-232, 2014.

20 Wang N, Chen C, Yang D, Liao Q, Luo H, Wang X, Zhou F, Yang X, Yang J, Zeng C and Wang WE: Mesenchymal stem cells-derived extracellular vesicles, via miR-210, improve infarcted cardiac function by promotion of angiogenesis. Biochim Biophys Acta 1863: 2085-2092, 2017.

21 Lou Y-L, Guo F, Liu F, Gao F-L, Zhang P-Q, Niu X, Guo S-C, Yin J-H, Wang Y and Deng Z-F: miR-210 activates notch signaling pathway in angiogenesis induced by cerebral ischemia. Mol Cell Biochem 370: 45-51, 2012.

22 Bakirtzi K, Law IKM, Xue X, Iliopoulos D, Shah YM and Pothoulakis C: Neurotensin promotes the development of colitis and intestinal angiogenesis via Hif- $1 \alpha-$ miR-210 signaling. J Immunol 196: 4311-4321, 2016.

23 Yang Y, Zhang J, Xia T, Li G, Tian T, Wang M, Wang R, Zhao L, Yang Y, Lan K and Zhou W: MicroRNA-210 promotes cancer angiogenesis by targeting fibroblast growth factor receptorlikeï ${ }_{i}^{1 / 21}$ in hepatocellular carcinoma. Oncol Rep 36: 2553-2562, 2016.

24 Xiao F, Qiu H, Zhou L, Shen X, Yang L and Ding K: WSS25 inhibits Dicer, downregulating microRNA-210, which targets Ephrin-A3, to suppress human microvascular endothelial cell (HMEC-1) tube formation. Glycobiology 23: 524-535, 2013.

25 Zhang S, Lai N, Liao K, Sun J and Lin Y: MicroRNA-210 regulates cell proliferation and apoptosis by targeting regulator of differentiation 1 in glioblastoma cells. Folia Neuropathol 53: 236-244, 2015.

26 Favaro E, Ramachandran A, McCormick R, Gee H, Blancher C, Crosby M, Devlin C, Blick C, Buffa F, Li J-L, Vojnovic B, Pires das Neves R, Glazer P, Iborra F, Ivan M, Ragoussis J and Harris AL: MicroRNA-210 regulates mitochondrial free radical response to hypoxia and krebs cycle in cancer cells by targeting iron sulfur cluster protein ISCU. PLoS One 5: e10345, 2010.

27 Li T, Song X, Zhang J, Zhao L, Shi Y, Li Z, Liu J, Liu N, Yan Y, Xiao Y, Tian X, Sun W, Guan Y and Liu B: Protection of human umbilical vein endothelial cells against oxidative stress by MicroRNA-210. Oxid Med Cell Longev 2017: 3565613, 2017.

28 Kosaka N, Sugiura K, Yamamoto Y, Yoshioka Y, Miyazaki H, Komatsu N, Ochiya $\mathrm{T}$ and Kato $\mathrm{T}$ : Identification of erythropoietin-induced microRNAs in haematopoietic cells during erythroid differentiation. Br J Haematol 142: 293-300, 2008.

29 Chen L, Zheng $\mathrm{H}$ and Zhang S: Involvement of up-regulation of miR-210 in a rat epilepsy model. Neuropsychiatr Dis Treat 12: 1731-1737, 2016.

30 Duan Z, Huang H and Sun F: The functional and predictive roles of miR-210 in cryptorchidism. Sci Rep 6: 32265, 2016.

31 Huang X, Le Q-T and Giaccia AJ: MiR-210 - micromanager of the hypoxia pathway. Trends Mol Med 16: 230-237, 2010.

32 Mathew LK and Simon MC: mir-210: A Sensor for Hypoxic Stress during Tumorigenesis. Mol Cell 35: 737-738, 2009. 
33 Ivan $\mathrm{M}$ and Huang $\mathrm{X}$ : miR-210: fine-tuning the hypoxic response. Adv Exp Med Biol 772: 205-227, 2014.

34 Huang X, Ding L, Bennewith KL, Tong RT, Welford SM, Ang KK, Story M, Le Q-T and Giaccia AJ: Hypoxia-inducible mir210 regulates normoxic gene expression involved in tumor initiation. Mol Cell 35: 856-867, 2009.

35 Qin Q, Furong W and Baosheng L: Multiple functions of hypoxia-regulated miR-210 in cancer. J Exp Clin Cancer Res 33: $50,2014$.

36 Dang K and Myers K: The Role of Hypoxia-induced miR-210 in cancer progression. Int J Mol Sci 16: 6353-6372, 2015.

37 Kulshreshtha R, Ferracin M, Wojcik SE, Garzon R, Alder H, Agosto-Perez FJ, Davuluri R, Liu C-G, Croce CM, Negrini M, Calin GA and Ivan M: A MicroRNA signature of hypoxia. Mol Cell Biol 27: 1859-1867, 2007.

38 Kelly TJ, Souza AL, Clish CB and Puigserver P: A hypoxiainduced positive feedback loop promotes hypoxia-inducible factor 1alpha stability through miR-210 suppression of glycerol3-phosphate dehydrogenase 1-like. Mol Cell Biol 31: 26962706, 2011

39 Costales MG, Haga CL, Velagapudi SP, Childs-Disney JL, Phinney DG and Disney MD: Small molecule inhibition of microRNA-210 reprograms an oncogenic hypoxic circuit. J Am Chem Soc 139: 3446-3455, 2017.

40 Ying Q, Liang L, Guo W, Zha R, Tian Q, Huang S, Yao J, Ding J, Bao M, Ge C, Yao M, Li J and He X: Hypoxia-inducible microRNA-210 augments the metastatic potential of tumor cells by targeting vacuole membrane protein 1 in hepatocellular carcinoma. Hepatology 54: 2064-2075, 2011.

41 Cheng HH, Mitchell PS, Kroh EM, Dowell AE, Chéry L, Siddiqui J, Nelson PS, Vessella RL, Knudsen BS, Chinnaiyan AM, Pienta KJ, Morrissey $\mathrm{C}$ and Tewari M: Circulating microRNA profiling identifies a subset of metastatic prostate cancer patients with evidence of cancer-associated hypoxia. PLoS One 8: e69239, 2013.

42 Crosby ME, Kulshreshtha R, Ivan M and Glazer PM: MicroRNA regulation of DNA repair gene expression in hypoxic stress. Cancer Res 69: 1221-1229, 2009.

43 Winther M, Alsner J, Sørensen BS, Wittrup CF, Tramm T, Baeksgaard L, Hofland K, Holtved E and Nordsmark M: Hypoxia-regulated MicroRNAs in gastroesophageal cancer. Anticancer Res 36: 721-730, 2016.

44 Sáenz-de-Santa-María I, Bernardo-Castiñeira C, Secades P, Bernaldo-de-Quirós S, Rodrigo JP, Astudillo A and Chiara M-D: Clinically relevant HIF-1 $\alpha$-dependent metabolic reprogramming in oropharyngeal squamous cell carcinomas includes coordinated activation of CAIX and the miR-210/ISCU signaling axis, but not MCT1 and MCT4 upregulation. Oncotarget 8: 13730-13746, 2017.

45 Yang W, Sun T, Cao J, Liu F, Tian Y and Zhu W: Downregulation of miR-210 expression inhibits proliferation, induces apoptosis and enhances radiosensitivity in hypoxic human hepatoma cells in vitro. Exp Cell Res 318: 944-954, 2012.

46 Zhu X, Han T, Sargent IL, Yin G and Yao Y: Differential expression profile of microRNAs in human placentas from preeclamptic pregnancies $v s$. normal pregnancies. Am J Obstet Gynecol 200: 661.e1-661.e7, 2009.

47 Fu G, Brkić J, Hayder H and Peng C: MicroRNAs in human placental development and pregnancy complications. Int J Mol Sci 14: 5519-5544, 2013.
48 Muralimanoharan S, Maloyan A, Mele J, Guo C, Myatt LG and Myatt L: MIR-210 modulates mitochondrial respiration in placenta with preeclampsia. Placenta 33: 816-823, 2012.

49 Luo R, Wang Y, Xu P, Cao G, Zhao Y, Shao X, Li Y, Chang C, Peng $\mathrm{C}$ and Wang Y: Hypoxia-inducible miR-210 contributes to preeclampsia via targeting thrombospondin type I domain containing 7A. Sci Rep 6: 19588, 2016.

50 Zhang Y, Fei M, Xue G, Zhou Q, Jia Y, Li L, Xin H and Sun S: Elevated levels of hypoxia-inducible microRNA-210 in preeclampsia: new insights into molecular mechanisms for the disease. J Cell Mol Med 16: 249-259, 2012.

51 Korkes HA, Oliveira L De, Sass N, Salahuddin S, Karumanchi SA and Rajakumar A: Relationship between hypoxia and downstream pathogenic pathways in preeclampsia. Hypertens Pregnancy: 1-6, 2017.

52 Kim JH, Park SG, Song S-Y, Kim JK and Sung J-H: Reactive oxygen species-responsive miR-210 regulates proliferation and migration of adipose-derived stem cells via PTPN2. Cell Death Dis 4: e588, 2013.

53 Adel S, Mansour A, Louka M, Matboli M, Elmekkawi SF and Swelam N: Evaluation of MicroRNA-210 and protein tyrosine phosphatase, non-receptor type 2 in Pre-eclampsia. Gene 596: 105-109, 2017.

54 Jung KO, Youn $\mathrm{H}$, Lee $\mathrm{C}-\mathrm{H}$, Kang $\mathrm{KW}$ and Chung $\mathrm{J}-\mathrm{K}$ : Visualization of exosome-mediated miR-210 transfer from hypoxic tumor cells. Oncotarget 8: 9899-9910, 2016.

55 Hsu Y-L, Hung J-Y, Chang W-A, Lin Y-S, Pan Y-C, Tsai P-H, Wu C-Y and Kuo P-L: Hypoxic lung cancer-secreted exosomal miR-23a increased angiogenesis and vascular permeability by targeting prolyl hydroxylase and tight junction protein $\mathrm{ZO}-1$. Oncogene 36: 4929-4942, 2017.

56 Hannafon BN and Ding W-Q: Intercellular communication by exosome-derived microRNAs in cancer. Int J Mol Sci 14: 14240-14269, 2013

57 King HW, Michael MZ and Gleadle JM: Hypoxic enhancement of exosome release by breast cancer cells. BMC Cancer 12: 421, 2012.

58 Zhang J, Li S, Li L, Li M, Guo C, Yao J and Mi S: Exosome and exosomal microRNA: trafficking, sorting, and function. Genomics Proteomics Bioinformatics 13: 17-24, 2015.

59 Jung E-J, Santarpia L, Kim J, Esteva FJ, Moretti E, Buzdar AU, Di Leo A, Le X-F, Bast RC, Park S-T, Pusztai L and Calin GA: Plasma microRNA 210 levels correlate with sensitivity to trastuzumab and tumor presence in breast cancer patients. Cancer 118: 2603-2614, 2012.

60 Qu A, Du L, Yang Y, Liu H, Li J, Wang L, Liu Y, Dong Z, Zhang $X$, Jiang $X$, Wang $H$, Li Z, Zheng $G$ and Wang C: Hypoxia-inducible MiR-210 is an independent prognostic factor and contributes to metastasis in colorectal cancer. PLoS One 9: e90952, 2014.

61 Wang W, Qu A, Liu W, Liu Y, Zheng G, Du L, Zhang X, Yang Y, Wang $C$ and Chen X: Circulating miR-210 as a diagnostic and prognostic biomarker for colorectal cancer. Eur J Cancer Care (Engl) 26, 2016. doi: 10.1111/ecc.12448

62 Sarakul O, Vattanaviboon P, Tanaka Y, Fucharoen S, Abe Y, Svasti $S$ and Umemura $\mathrm{T}$ : Enhanced erythroid cell differentiation in hypoxic condition is in part contributed by miR-210. Blood Cells Mol Dis 51: 98-103, 2013.

63 Bianchi N, Zuccato C, Lampronti I, Borgatti M and Gambari R: Expression of miR-210 during erythroid differentiation and 
induction of gamma-globin gene expression. BMB Rep 42: 493-499, 2009.

64 Bavelloni A, Poli A, Fiume R, Blalock W, Matteucci A, Ramazzotti G, McCubrey JA, Cocco L and Faenza I: PLC-beta 1 regulates the expression of miR-210 during mithramycinmediated erythroid differentiation in K562 cells. Oncotarget 5: 4222-4231, 2014.

65 Faenza I and Cocco L: Emerging role for PLC $\beta 1$ MiRNA and disease. Rna Dis: 1-6, 2015.

66 Siwaponanan P, Fucharoen S, Sirankapracha P, Winichagoon P, Umemura T and Svasti S: Elevated levels of miR-210 correlate with anemia in $\beta$-thalassemia/HbE patients. Int J Hematol 104: 338-343, 2016.

67 Li L, Huang K, You Y, Fu X, Hu L, Song L and Meng Y: Hypoxia-induced miR-210 in epithelial ovarian cancer enhances cancer cell viability via promoting proliferation and inhibiting apoptosis. Int J Oncol 44: 2111-2120, 2014.

68 Ding L, Zhao L, Chen W, Liu T, Li Z and Li X: miR-210, a modulator of hypoxia-induced epithelial-mesenchymal transition in ovarian cancer cell. Int J Clin Exp Med 8: 2299-2307, 2015.

69 Cicchillitti L, Di Stefano V, Isaia E, Crimaldi L, Fasanaro P, Ambrosino V, Antonini A, Capogrossi MC, Gaetano C, Piaggio $\mathrm{G}$ and Martelli F: Hypoxia-inducible factor 1- $\alpha$ induces miR210 in normoxic differentiating myoblasts. J Biol Chem 287: 44761-44771, 2012.

70 Mutharasan RK, Nagpal V, Ichikawa Y and Ardehali H: microRNA-210 is up-regulated in hypoxic cardiomyocytes through Akt- and p53-dependent pathways and exerts cytoprotective effects. AJP Hear Circ Physiol 301: H1519H1530, 2011.

71 Yang W, Wei J, Guo T, Shen Y and Liu F: Knockdown of miR210 decreases hypoxic glioma stem cells stemness and radioresistance. Exp Cell Res 326: 22-35, 2014.

72 Acunzo M, Romano G, Wernicke D and Croce CM: MicroRNA and cancer - A brief overview. Adv Biol Regul 57: 1-9, 2015.

73 Malzkorn B, Wolter M, Liesenberg F, Grzendowski M, Stühler $\mathrm{K}$, Meyer $\mathrm{HE}$ and Reifenberger G: Identification and functional characterization of microRNAs involved in the malignant progression of gliomas. Brain Pathol 20: 539-550, 2010.

74 Satzger I, Mattern A, Kuettler U, Weinspach D, Voelker B, Kapp A and Gutzmer R: MicroRNA-15b represents an independent prognostic parameter and is correlated with tumor cell proliferation and apoptosis in malignant melanoma. Int $\mathrm{J}$ cancer 126: 2553-2562, 2010.

75 Foekens JA, Sieuwerts AM, Smid M, Look MP, de Weerd V, Boersma AWM, Klijn JGM, Wiemer EAC and Martens JWM: Four miRNAs associated with aggressiveness of lymph nodenegative, estrogen receptor-positive human breast cancer. Proc Natl Acad Sci USA 105: 13021-13026, 2008.

76 Greither T, Grochola LF, Udelnow A, Lautenschläger C, Würl P and Taubert H: Elevated expression of microRNAs 155, 203, 210 and 222 in pancreatic tumors is associated with poorer survival. Int J cancer 126: 73-80, 2010.

77 Camps C, Buffa FM, Colella S, Moore J, Sotiriou C, Sheldon $\mathrm{H}$, Harris AL, Gleadle JM and Ragoussis J: hsa-miR-210 Is induced by hypoxia and is an independent prognostic factor in breast cancer. Clin Cancer Res 14: 1340-1348, 2008.

78 Zhang Z, Sun H, Dai H, Walsh RM, Imakura M, Schelter J, Burchard J, Dai X, Chang AN, Diaz RL, Marszalek JR, Bartz SR, Carleton M, Cleary MA, Linsley PS and Grandori C:
MicroRNA miR-210 modulates cellular response to hypoxia through the MYC antagonist MNT. Cell Cycle 8: 2756-2768, 2009.

79 Wang J, Zhao J, Shi M, Ding Y, Sun H, Yuan F and Zou Z: Elevated Expression of miR-210 Predicts Poor Survival of Cancer Patients: A Systematic Review and Meta-Analysis. PLoS One 9: e89223, 2014.

80 Radojicic J, Zaravinos A, Vrekoussis T, Kafousi M, Spandidos DA and Stathopoulos EN: MicroRNA expression analysis in triple-negative (ER, PR and Her2/neu) breast cancer. Cell Cycle 10: 507-517, 2011.

81 Toyama T, Kondo N, Endo Y, Sugiura H, Yoshimoto N, Iwasa M, Takahashi S, Fujii Y and Yamashita H: High expression of MicroRNA-210 is an independent fctor indicating a poor prognosis in Japanese triple-negative breast cancer patients. Jpn J Clin Oncol 42: 256-263, 2012.

82 Volinia S, Galasso M, Sana ME, Wise TF, Palatini J, Huebner K and Croce CM: Breast cancer signatures for invasiveness and prognosis defined by deep sequencing of microRNA. Proc Natl Acad Sci 109: 3024-3029, 2012.

83 Bastos EP, Brentani H, Pasini FS, Silva ART, Torres CH, Puga RD, Olivieri EHR, Piovezani AR, Pereira CA de B, MachadoLima A, Carraro DM and Brentani MM: MicroRNAs discriminate familial from sporadic Non-BRCA1/2 breast carcinoma arising in patients $\leq 35$ years. PLoS One 9: e101656, 2014.

84 Rothé F, Ignatiadis M, Chaboteaux C, Haibe-Kains B, Kheddoumi N, Majjaj S, Badran B, Fayyad-Kazan H, Desmedt C, Harris AL, Piccart M and Sotiriou C: Global microRNA expression profiling identifies MiR-210 associated with tumor proliferation, invasion and poor clinical outcome in breast cancer. PLoS One 6: e20980, 2011.

85 Camps C, Buffa FM, Colella S, Moore J, Sotiriou C, Sheldon $\mathrm{H}$, Harris AL, Gleadle JM and Ragoussis J: hsa-miR-210 is induced by hypoxia and is an independent prognostic factor in breast cancer. Clin Cancer Res 14: 1340-1348, 2008.

86 Bar I, Merhi A, Abdel-Sater F, Ben Addi A, Sollennita S, Canon J-L and Delrée P: The MicroRNA miR-210 is expressed by cancer cells but also by the tumor microenvironment in triple-negative breast cancer. J Histochem Cytochem: 2215541770284, 2017.

87 Devlin C, Greco S, Martelli F and Ivan M: miR-210: More than a silent player in hypoxia. IUBMB Life 63: 94-100, 2011.

88 Puisségur M-P, Mazure NM, Bertero T, Pradelli L, Grosso S, Robbe-Sermesant K, Maurin T, Lebrigand K, Cardinaud B, Hofman V, Fourre S, Magnone V, Ricci JE, Pouysségur J, Gounon P, Hofman P, Barbry P and Mari B: miR-210 is overexpressed in late stages of lung cancer and mediates mitochondrial alterations associated with modulation of HIF-1 activity. Cell Death Differ 18: 465-478, 2011.

89 Sundarbose K, Kartha R and Subramanian S: MicroRNAs as Biomarkers in Cancer. Diagnostics 3: 84-104, 2013.

90 Larrea E, Sole C, Manterola L, Goicoechea I, Armesto M, Arestin M, Caffarel M, Araujo A, Araiz M, Fernandez-Mercado M and Lawrie C: New concepts in cancer biomarkers: circulating miRNAs in liquid biopsies. Int J Mol Sci 17: 627, 2016.

91 Simpson RJ, Lim JW, Moritz RL and Mathivanan S: Exosomes: proteomic insights and diagnostic potential. Expert Rev Proteomics 6: 267-283, 2009.

92 Matsuzaki J and Ochiya T: Circulating microRNAs and extracellular vesicles as potential cancer biomarkers: a systematic review. Int J Clin Oncol 22: 413-420, 2017. 
93 Zhao A, Li G, Péoc'h M, Genin C and Gigante M: Serum miR210 as a novel biomarker for molecular diagnosis of clear cell renal cell carcinoma. Exp Mol Pathol 94: 115-120, 2013.

94 Li G, Zhao A, Péoch M, Cottier M and Mottet N: Detection of urinary cell-free miR-210 as a potential tool of liquid biopsy for clear cell renal cell carcinoma. Urol Oncol Semin Orig Investig 35: 294-299, 2017.

95 McCormick RI, Blick C, Ragoussis J, Schoedel J, Mole DR, Young AC, Selby PJ, Banks RE and Harris AL: miR-210 is a target of hypoxia-inducible factors 1 and 2 in renal cancer, regulates ISCU and correlates with good prognosis. Br J Cancer 108: 1133-1142, 2013.

96 Yoshino H, Yonemori M, Miyamoto K, Tatarano S, Kofuji S, Nohata N, Nakagawa $M$ and Enokida H: microRNA-210-3p depletion by CRISPR/Cas 9 promoted tumorigenesis through revival of TWIST1 in renal cell carcinoma. Oncotarget 8 : 20881-20894, 2017.

97 Yang Y, Qu A, Liu J, Wang R, Liu Y, Li G, Duan W, Fang Q, Jiang X, Wang L, Zheng G, Du L, Zhang X and Wang C: Serum miR-210 contributes to tumor detection, stage prediction and dynamic surveillance in patients with bladder cancer. PLoS One 10: $\mathrm{e} 0135168,2015$.

98 Dong F, Xu T, Shen Y, Zhong S, Chen S, Ding Q and Shen Z: Dysregulation of miRNAs in bladder cancer: altered expression with aberrant biogenesis procedure. Oncotarget 8: 27547-27568, 2017.

99 Cai H, Lin L, Cai H, Tang M and Wang Z: Prognostic evaluation of microRNA-210 expression in pediatric osteosarcoma. Med Oncol 30: 499, 2013.

100 Koperski L, Kotlarek M, Swierniak M, Kolanowska M, Kubiak A, Gornicka B, Jazdzewski K and Wojcicka A: Next-generation sequencing reveals microRNA markers of adrenocortical tumors malignancy. Oncotarget 8: 49191-49200, 2017.

101 Liu C and Tang X: Down-regulation of microRNA-210 inhibits osteosarcoma growth in vitro and in vivo. Mol Med Rep 12: 3674-3680, 2015.

102 Zhang H, Mai Q and Chen J: MicroRNA-210 is increased and it is required for dedifferentiation of osteosarcoma cell line. Cell Biol Int 41: 267-275, 2017.

103 Tsuchiya S, Fujiwara T, Sato F, Shimada Y, Tanaka E, Sakai Y, Shimizu K and Tsujimoto G: MicroRNA-210 regulates cancer cell proliferation through targeting fibroblast growth factor receptor-like 1 (FGFRL1). J Biol Chem 286: 420-428, 2011.

104 Zuo J, Wen M, Lei M, Peng X, Yang X and Liu Z: MiR-210 links hypoxia with cell proliferation regulation in human Laryngocarcinoma cancer. J Cell Biochem 116: 1039-1049, 2015.

105 Giannakakis A, Sandaltzopoulos R, Greshock J, Liang S, Huang J, Hasegawa K, Li C, O'Brien-Jenkins A, Katsaros D, Weber BL, Simon C, Coukos G and Zhang L: miR-210 links hypoxia with cell cycle regulation and is deleted in human epithelial ovarian cancer. Cancer Biol Ther 7: 255-264, 2008.

106 Biswas S, Roy S, Banerjee J, Hussain S-RA, Khanna S, Meenakshisundaram G, Kuppusamy P, Friedman A and Sen CK: Hypoxia inducible microRNA 210 attenuates keratinocyte proliferation and impairs closure in a murine model of ischemic wounds. Proc Natl Acad Sci USA 107: 6976-6981, 2010.
$107 \mathrm{He}$ J, Wu J, Xu N, Xie W, Li M, Li J, Jiang Y, Yang BB and Zhang Y: MiR-210 disturbs mitotic progression through regulating a group of mitosis-related genes. Nucleic Acids Res 41: 498-508, 2013.

108 Nakada C, Tsukamoto Y, Matsuura K, Nguyen TL, Hijiya N, Uchida T, Sato F, Mimata H, Seto $M$ and Moriyama M: Overexpression of miR-210, a downstream target of HIF1 $\alpha$, causes centrosome amplification in renal carcinoma cells. J Pathol 224: 280-288, 2011.

109 Phuah NH, Azmi MN, Awang K and Nagoor NH: Downregulation of MicroRNA-210 confers sensitivity towards 1'S-1'Acetoxychavicol Acetate (ACA) in cervical cancer cells by targeting SMAD4. Mol Cells 40: 291-298, 2017.

110 Lai N, Dong Q, Ding H, Miao Z and Lin Y: MicroRNA-210 overexpression predicts poorer prognosis in glioma patients. J Clin Neurosci 21: 755-760, 2014.

111 Lai N, Zhu H, Chen Y, Zhang S, Zhao X and Lin Y: Differential expression of microRNA-210 in gliomas of variable cell origin and correlation between increased expression levels and disease progression in astrocytic tumours. Folia Neuropathol 52: 79-85, 2014.

112 Li Y, Dai Y, Zhang X and Chen J: Three-layered polyplex as a microRNA targeted delivery system for breast cancer gene therapy. Nanotechnology 28: 285101, 2017.

113 Kulshreshtha R, Davuluri R V, Calin GA and Ivan M: A microRNA component of the hypoxic response. Cell Death Differ 15: 667-671, 2008.

114 Greco S, Gaetano C and Martelli F: HypoxamiR regulation and function in ischemic cardiovascular diseases. Antioxid Redox Signal 21: 1202-1219, 2014.

115 Mok Y, Schwierzeck V, Thomas DC, Vigorito E, Rayner TF, Jarvis LB, Prosser HM, Bradley A, Withers DR, Mårtensson IL, Corcoran LM, Blenkiron C, Miska EA, Lyons PA and Smith KGC: MiR-210 is induced by Oct-2, regulates B cells, and inhibits autoantibody production. J Immunol 191: 3037-3048, 2013.

116 Muralimanoharan S, Guo C, Myatt L and Maloyan A: Sexual dimorphism in miR-210 expression and mitochondrial dysfunction in the placenta with maternal obesity. Int J Obes 39: 1274-1281, 2015.

117 Mutharasan RK, Nagpal V, Ichikawa Y and Ardehali H: microRNA-210 is upregulated in hypoxic cardiomyocytes through Akt- and p53-dependent pathways and exerts cytoprotective effects. Am J Physiol Heart Circ Physiol 301: H1519-1530, 2011.

118 Grün D, Wang Y-L, Langenberger D, Gunsalus KC and Rajewsky N: microRNA target predictions across seven Drosophila species and comparison to mammalian targets. PLoS Comput Biol 1: e13, 2005.
Received September 14, 2017

Revised September 27, 2017

Accepted September 28, 2017 